\title{
ON THE MINIMUM VECTOR RANK OF MULTIGRAPHS*
}

\author{
LON H. MITCHELL ${ }^{\dagger}$, SIVARAM K. NARAYAN ${ }^{\ddagger}$, AND IAN ROGERS $\$$
}

\begin{abstract}
The minimum vector rank (mvr) of a graph over a field $\mathbb{F}$ is the smallest $d$ for which a faithful vector representation of $G$ exists in $\mathbb{F}^{d}$. For simple graphs, minimum semidefinite rank (msr) and minimum vector rank differ by exactly the number of isolated vertices. We explore the relationship between msr and mvr for multigraphs and show that a result linking the msr of chordal graphs to clique cover number also holds for the mvr of multigraphs. We study the difference between msr and mvr in the removal of duplicate vertices in multigraphs, and relate mvr to certain coloring problems.
\end{abstract}

Key words. Minimum semidefinite rank, Minimum vector rank, Duplicate vertices, Chordal graph.

AMS subject classifications. 15A18, 05C50, 15A57.

1. Introduction. A graph $G$ consists of a set of vertices $V(G)$ and a set of edges $E(G)$ which are unordered pairs of vertices. A graph is simple if it has no multiple edges or loops. In what follows, we only consider graphs that have no loops, but may have multiple edges (multigraphs).

A subset of vertices and the set of edges among them in $G$ form an induced subgraph of $G$. We let $N(v)$ denote the neighborhood of a vertex $v$, which is the set of vertices of $G$ adjacent to $v$, and set $N[v]=\{v\} \cup N(v)$. We also define $N_{1}(v)$ to be the set of vertices of $G$ adjacent to $v$ by exactly one edge, and set $N_{1}[v]=\{v\} \cup N_{1}(v)$. Note that, for a simple graph, $N_{1}(v)=N(v)$ for every vertex $v$. We say a vertex $v$ is singly-isolated if $N_{1}(v)$ is empty.

A square matrix $M=\left[m_{i j}\right]$ is combinatorially symmetric when $m_{i j}=0$ if and only if $m_{j i}=0$. If $G$ is a graph on $n$ vertices that has no loops, let $\mathcal{C}(G, \mathbb{F})$ denote the set of all $n$-by- $n$ combinatorially symmetric matrices with entries in the field $\mathbb{F}$ such that

${ }^{*}$ Received by the editors October 28, 2009. Accepted for publication August 30, 2010. Handling Editor: Raphael Loewy.

${ }^{\dagger}$ Department of Mathematics, Virginia Commonwealth University, Richmond, VA 23284 (lmitchell2@vcu.edu).

$\ddagger$ Department of Mathematics, Central Michigan University, Mt. Pleasant, MI 48859 (sivaram.narayan@cmich.edu). Research supported in part by NSF grant DMS 05-52594. This author thanks Central Michigan University for its support during his Fall 2009 sabbatical leave and Virginia Commonwealth University for the hospitality extended during his sabbatical visit when this work was completed.

$\S$ Department of Mathematics, Rose-Hulman Institute of Technology, Terre Haute, IN 47803. Research supported in part by NSF grant DMS 05-52594. 
- $a_{i j} \neq 0$ if $i$ and $j$ are joined by exactly one edge, and

- $a_{i j}=0$ if $i \neq j$ and $i$ and $j$ are not adjacent.

Minimum rank problems seek to find the minimum rank over matrices in a given subset of $\mathcal{C}(G, \mathbb{F})$ for a specified $G$ and $\mathbb{F}$. For positive semidefinite (psd) matrices, this is the minimum semidefinite rank, $\operatorname{mr}_{+}(G)$ for $\mathbb{F}=\mathbb{R}$ and $\operatorname{msr}(G)$ for $\mathbb{F}=\mathbb{C}$. This problem has been previously studied both for multigraphs as we have presented it $[1,4,7,19]$ and when the graph $G$ is required to be simple $[3,11]$.

We say $\vec{X}=\left\{\vec{x}_{1}, \ldots, \vec{x}_{n}\right\} \subset \mathbb{F}^{m}$ is an (orthogonal) vector representation [16] of $G$ when $X^{*} X \in \mathcal{C}(G, \mathbb{F})$, where $X$ is the matrix whose columns are the vectors $\vec{x}_{i}$. In this paper, $\mathbb{F}$ will be either $\mathbb{R}$ or $\mathbb{C}$, and we will only consider the class of psd matrices over $\mathbb{F}$. By the rank of a vector representation, we mean the dimension of the span of the vectors.

Positive semidefinite matrices in $M_{n}(\mathbb{F})$ may be characterized by their factorization as $X^{*} X$ for some $X \in M_{n}(\mathbb{F})$ of the same rank so that each psd matrix is a Gram matrix of a certain set of vectors. Therefore, the smallest $m$ for which there exists a vector representation of $G$ in $\mathbb{F}^{m}$ is equal to the msr, and finding a psd matrix with a given graph and finding a vector representation of the graph are equivalent problems.

Given a vector representation $\vec{V}$ of a graph $G$, for a fixed vertex $v$, we may "orthogonally remove" the vector $\vec{v}$ that corresponds to $v$ by orthogonally projecting each vector of $\vec{V}$ onto the complement of the span of $\vec{v}$. This yields a vector representation $\vec{V} \ominus \vec{v}$ with rank decreased by one of a graph $G^{\prime}$ with order decreased by one. We define $G \ominus v$ so that $\vec{V} \ominus \vec{v}$ is a vector representation of $G \ominus v$ as follows: in the induced subgraph $G-v$ of $G$, between any $u, w \in N(v)$ add $e-1$ edges, where $e$ is the sum of the number of edges between $u$ and $v$ and the number of edges between $w$ and $v$. By construction, $\operatorname{msr}(G) \geq \operatorname{msr}(G \ominus v)+1$.

We will say that a multigraph is complete if $N[v]=V(G)$ for every vertex $v$. Recall that a graph is chordal if it does not contain an induced subgraph that is a cycle on four or more vertices, a clique is a maximal induced complete subgraph, and that a vertex $v$ is simplicial if $N[v]$ is a clique. Further, every chordal graph has a simplicial vertex [2, pg. 175]. The clique number of a graph $G, \omega(G)$, is the order of a largest clique of $G$ [20].

In the original definition, vector representations of a graph may include a zero vector. Thus, isolated and singly-isolated vertices do not influence the msr of a graph. In certain situations, such as computing the msr of the join of two graphs [7], it has been beneficial to consider only non-degenerate vector representations that do not include zero vectors. This led to the definition of the minimum vector rank (mvr) 
of a graph $G$ as the minimum rank among non-degenerate vector representations of $G$.

For simple graphs, mvr and msr differ by exactly the number of isolated vertices. Therefore, if $G$ is a connected simple graph on two or more vertices and $H$ is an induced subgraph of $G$, then $\operatorname{mvr}(G)=\operatorname{msr}(G) \geq \operatorname{mvr}(H)$ [11, Proposition 2.3]. For connected multigraphs, this need not be the case, and the relationship between mvr and msr is not known. For example, let $G$ be a path on three vertices with the single edges replaced by double edges and let $\mathrm{H}$ be the complete graph on two vertices with the single edge replaced by a double edge. Then $\operatorname{msr}(G)=0$ but $\operatorname{mvr}(H)=1$.

For a graph $G$, let $\mathcal{S}(G)$ denote the set of simple (not necessarily connected) subgraphs of $G$ (so that $H \in \mathcal{S}(G)$ is obtained from $G$ by repeatedly either deleting a multiple edge or replacing a multiple edge by a single edge).

For a multigraph $G$, we say that a set of subgraphs $G_{1}, \ldots, G_{n}$ is a vertex cover of $G$ if each vertex of $G$ is a vertex of at least one $G_{i}$, and an edge cover if for every pair of vertices $v$ and $w$ of $G$ that are adjacent by exactly one edge in $G$, there is at least one $G_{i}$ in which $v$ and $w$ are adjacent by exactly one edge. An edge or vertex cover by simple cliques is called a clique cover. These definitions extend the usual ones for simple graphs.

Clique covers of graphs have been studied in many different contexts, and have a corresponding number of different notations. We will use $\theta(G)=\theta_{0}(G)$ for the vertex clique cover number, $\mathrm{cc}(G)=\theta_{1}(G)$ for the edge clique cover number, and define $\theta_{2}(G)$ to be the smallest possible number of cliques in a clique cover that is both a vertex and edge cover (cf. $[5,17,18]$ ).

For a simple graph, it is well known that edge covers give upper bounds on msr. In the case of multigraphs and mvr, this remains true. Except for the final claim, the following lemma has appeared previously in the case of $\mathbb{F}=\mathbb{C}$ [4, Lemma 3.3], but we include a proof of all claims for completeness.

Lemma 1.1. Let $\mathbb{F}=\mathbb{C}$ or $\mathbb{F}=\mathbb{R}$. Suppose that $\vec{X}_{1}, \ldots, \vec{X}_{m}$, with $\vec{X}_{i} \subseteq \mathbb{F}^{n}$ for $1 \leq i \leq m$, are vector representations of subgraphs $G_{1}, \ldots, G_{m}$ of a multigraph $G$ such that

- $G_{1}, \ldots, G_{m}$ is an edge cover of $G$, and

- for every pair of vertices $v$ and $w$ that are not adjacent in $G$, if $\vec{x}_{v}$ represents vertex $v$ in $\vec{X}_{i}$ and $\vec{x}_{w}$ represents vertex $w$ in $\vec{X}_{j}$, then $\vec{x}_{v}$ and $\vec{x}_{w}$ are orthogonal, 
then there exists a vector representation $\vec{X}$ of $G$ with

$$
\operatorname{rank} \vec{X} \leq \operatorname{rank}\left(\operatorname{Span} \bigcup_{i=1}^{m} \vec{X}_{i}\right) \leq \sum_{i=1}^{m} \operatorname{rank} \vec{X}_{i} .
$$

Further, if for every vertex $v$ of $G$, there exists an $i$ such that the vector representing $v$ in $\vec{X}_{i}$ is non-zero, then $\vec{X}$ will be non-degenerate.

Proof. We prove the statement for the case of two vector representations as that result can be used repeatedly to give the more general case. Let $\vec{X}=\left\{\vec{x}_{i}\right\}$ and $\vec{W}=\left\{\vec{w}_{i}\right\}$ be vector representations of subgraphs $G_{1}$ and $G_{2}$ of a graph $G$. Extend $\vec{X}$ and $\vec{W}$ to represent all vertices of $G$ by adding copies of the zero vector if need be. We claim there exists a scalar $c \in \mathbb{F}$ such that $\left\{\vec{x}_{i}+c \vec{w}_{i}\right\}$ is a vector representation of $G$.

If vertices $v_{i}$ and $v_{j}$ are not adjacent in $G$, then the inner products $\left\langle\vec{x}_{i}, \vec{x}_{j}\right\rangle$, $\left\langle\vec{x}_{i}, \vec{w}_{j}\right\rangle,\left\langle\vec{w}_{i}, \vec{w}_{j}\right\rangle$, and $\left\langle\vec{w}_{i}, \vec{x}_{j}\right\rangle$ are all zero. Then $\left\langle\vec{x}_{i}+c \vec{w}_{i}, \vec{x}_{j}+c \vec{w}_{j}\right\rangle$ is also zero for any choice of $c$. If $v_{i}$ and $v_{j}$ are adjacent by exactly one edge in $G$, then $\left\{\left\langle\vec{x}_{i}+c \vec{w}_{i}, \vec{x}_{j}+c \vec{w}_{j}\right\rangle\right\}$ is a set of quadratic polynomials in $c$ having finitely many roots. Thus there exists a $c \in \mathbb{F}$ such that $\left\{\vec{x}_{i}+c \vec{w}_{i}\right\}$ is a vector representation of $G$.

If for every vertex $v_{i}$ of $G$, either $\vec{x}_{i}$ or $\vec{w}_{i}$ is non-zero, there are at most finitely many $c$ for which some $\vec{x}_{i}+c \vec{w}_{i}$ will be the zero vector. Thus, in this case, there exists a $c \in \mathbb{F}$ such that $\left\{\vec{x}_{i}+c \vec{w}_{i}\right\}$ is a non-degenerate vector representation of $G$. $\square$

2. Duplicate Vertices. For a simple graph $G$, we say a vertex $v$ is a duplicate of a vertex $w$ if $N[v]=N[w]$. Since duplicate vertices may be represented by the same vector in a vector representation of $G$, we have

Theorem 2.1 ([4, Proposition 2.2]). Let $G$ be a simple connected graph on three or more vertices. If $u$ is a duplicate of a vertex $v$ in $G$, then $\operatorname{msr}(G)=\operatorname{msr}(G-u)$.

As shown in Example 2.2, for a graph $G$ with multiple edges, the result of Theorem 2.1 may no longer be true when trying to use the same definition, since, even if $N[v]=N[w]$ in $G$, the closed neighborhoods of $v$ and $w$ need not be the same in a graph of $\mathcal{S}(G)$.

ExAmple 2.2. In Figure 2.1, $N[v]=N[w]$ in $G$, and removing $w$ leaves the graph $G-w$ which has msr two, given by $H \in \mathcal{S}(G-w)$. However, every graph in $\mathcal{S}(G)$ (and therefore $G$ ) has msr three.

As shown in Example 2.2, in the multigraph setting, for $v$ and $w$ to act like duplicates, it is not even enough for there to exist a graph $H$ in $\mathcal{S}(G)$ with $\operatorname{msr}(H)=$ $\operatorname{msr}(G)$ where $v$ and $w$ are duplicates in $H$. 


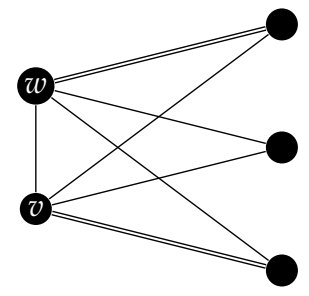

G

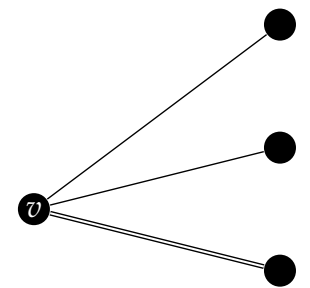

$G-w$

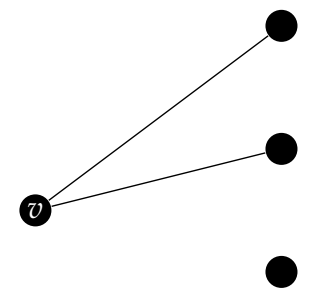

$H$

FIG. 2.1. Graphs to accompany Example 2.2.

For a simple graph $G$, if there exists a vector representation of $G$ in which two vertices $v$ and $w$ are represented by parallel vectors, then $v$ and $w$ must be duplicate vertices. Example 2.2 also shows that the existence of a vector representation of a multigraph in which two vertices are represented by parallel vectors, and even such a vector representation that is minimal with respect to rank, does not guarantee that the removal of one vertex will not affect msr.

Instead, we suggest that if $v$ and $w$ are adjacent in $G$ and satisfy $N_{1}(w) \subseteq N_{1}[v]$ and $N[v] \subseteq N[w]$, we will say that $w$ duplicates $v$. If $w$ duplicates $v$ and $v$ duplicates $w$, then $v$ and $w$ are duplicate vertices. If the graph is simple, then $v$ and $w$ are duplicate vertices in the original sense. Moreover, in a simple graph, since $N_{1}(w)=$ $N(w), w$ duplicates $v$ if and only if $v$ and $w$ are duplicate vertices.

Remark 2.3. From the definition, if $w$ duplicates $v$, then $w$ and $v$ are adjacent. Also, if $v$ is singly-isolated then so is $w$.

Proposition 2.4. Let $G$ be a multigraph containing vertices $v$ and $w$ where $w d u$ plicates $v$. Then $\operatorname{msr}(G) \leq \operatorname{msr}(G-w)+1$. If further $N_{1}(v) \neq\{w\}$, then $\operatorname{msr}(G)=$ $\operatorname{msr}(G-w)$.

Proof. Since $G-w$ is an induced subgraph of $G, \operatorname{msr}(G) \geq \operatorname{msr}(G-w)$. If $w$ is singly-isolated in $G$, we are done, so we may assume that neither $v$ nor $w$ are singly-isolated.

First, suppose that $N_{1}(v) \neq\{w\}$. In order to establish that $\operatorname{msr}(G) \leq \operatorname{msr}(G-$ $w)$, since the desired result holds for simple graphs, our strategy will be to choose simple graph representatives of $G$ and $G-w$ to work with: let $H^{\prime}$ be any graph in $\mathcal{S}(G-w)$ such that $\operatorname{msr}\left(H^{\prime}\right)=\operatorname{msr}(G-w)$ and take $H$ to be the unique graph in $\mathcal{S}(G)$ such that $v$ and $w$ are duplicate vertices in $H$ and $H-w=H^{\prime}$. Because $N_{1}(v) \neq\{w\}$, the connected component of $H$ containing $v$ and $w$ has at least three vertices. Therefore, Theorem 2.1 may be applied to $H$ and $H-w$ to yield $\operatorname{msr}(G) \leq$ 


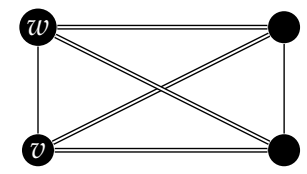

$G_{1}$

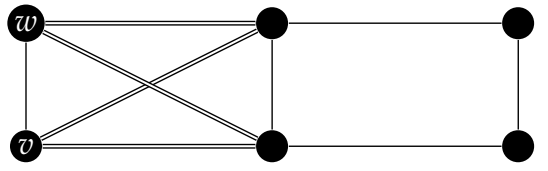

$G_{2}$

Fig. 2.2. Graphs to accompany Example 2.5.

$\operatorname{msr}(H)=\operatorname{msr}(H-w)=\operatorname{msr}(G-w)$.

For the remaining case, we will again resort to working with simple graph representatives: if $N_{1}(v)=\{w\}$, then $v$ is singly-isolated in $G-w$ so that $\operatorname{msr}(G-$ $w)=\operatorname{msr}(G-w-v)$. If $H \in \mathcal{S}(G-w-v)$ with $\operatorname{msr}(H)=\operatorname{msr}(G-w-v)$, then adding a $K_{2}$ component on two vertices $v$ and $w$ to $H$ gives a graph $G^{\prime}$ with $G^{\prime} \in \mathcal{S}(G)$, and $\operatorname{msr}\left(G^{\prime}\right)=\operatorname{msr}(H)+1$. Thus

$$
\operatorname{msr}(G) \leq \operatorname{msr}\left(G^{\prime}\right)=\operatorname{msr}(H)+1=\operatorname{msr}(G-w-v)+1=\operatorname{msr}(G-w)+1 . \square
$$

Example 2.5. Referring to Figure 2.2, in both $G_{1}$ and $G_{2}, w$ duplicates $v$ and $N_{1}(v)=\{w\}$. Further, $\operatorname{msr}\left(G_{1}\right)=1=\operatorname{msr}\left(G_{1}-w\right)$, but $\operatorname{msr}\left(G_{2}\right)=3$ while $\operatorname{msr}\left(G_{2}-w\right)=2$, showing that the inequality in Proposition 2.4 can be strict.

If $v$ is simplicial, we can get a result similar to Proposition 2.4 under less restrictive assumptions.

Proposition 2.6. Let $v$ be a simplicial vertex of a multigraph $G$ that is not singlyisolated, $w$ a neighbor of $v$, and $N_{1}[w] \subseteq N[v]$. Then $\operatorname{msr}(G)=\operatorname{msr}(G-w)$.

Proof. Because $G-w$ is an induced subgraph of $G, \operatorname{msr}(G) \geq \operatorname{msr}(G-w)$. Take a vector representation $\vec{X}_{1}$ of $G-w$ with $\operatorname{rank}\left(\vec{X}_{1}\right)=\operatorname{msr}(G-w)$, and let $G_{1}$ be the corresponding simple graph in $\mathcal{S}(G-w)$ such that $\operatorname{msr}\left(G_{1}\right)=\operatorname{msr}(G-w)$. Let $\vec{v}$ be the vector of $\vec{X}_{1}$ representing vertex $v$, and let $G_{2}$ be the simple complete graph on the vertices of $N[v]$ in $G$. Define a vector representation $\vec{X}_{2}$ of $G_{2}$ by letting every vector be represented by $\vec{v}$. Because $v$ is simplicial in $G, G_{1}$ and $G_{2}$ along with $\vec{X}_{1}$ and $\vec{X}_{2}$ satisfy the conditions of Lemma 1.1, and so there exists a vector representation $\vec{X}$ of $G$ with

$$
\operatorname{rank}(\vec{X}) \leq \operatorname{rank}\left(\operatorname{Span}\left(\vec{X}_{1} \cup \vec{X}_{2}\right)\right)=\operatorname{rank}\left(\operatorname{Span} \vec{X}_{1}\right)=\operatorname{msr}(G-w)
$$

showing that $\operatorname{msr}(G) \leq \operatorname{msr}(G-w)$. 
Duplicate vertices behave somewhat better with respect to mvr than they do with respect to msr:

Proposition 2.7. Let $G$ be a simple graph with duplicate vertices $v$ and $w$. Then $\operatorname{mvr}(G)=\operatorname{mvr}(G-w)$.

Proof. Since $G-w$ is an induced subgraph of $G, \operatorname{mvr}(G) \geq \operatorname{mvr}(G-w)$. Let $\vec{X}$ be a non-degenerate vector representation of $G-w$, and let $\vec{v}$ be the vector representing $v$ in $\vec{X}$. Then, setting $\vec{w}=\vec{v}, \vec{X}^{\prime}=\vec{X} \cup\{\vec{w}\}$ is a non-degenerate vector representation of $G$ with $\operatorname{rank} \vec{X}^{\prime}=\operatorname{rank} \vec{X}$, showing $\operatorname{mvr}(G) \leq \operatorname{mvr}(G-w)$.

Proposition 2.8. Let $G$ be a multigraph containing vertices $v$ and $w$ where $w$ duplicates $v$. Then $\operatorname{mvr}(G)=\operatorname{mvr}(G-w)$.

Proof. Let $v$ and $w$ be adjacent in $G, N_{1}(w) \subseteq N_{1}[v]$, and $N[v] \subseteq N[w]$. Let $H^{\prime}$ be any graph in $\mathcal{S}(G-w)$ such that $\operatorname{mvr}\left(H^{\prime}\right)=\operatorname{mvr}(G-w)$. Take $H$ to be the unique graph in $\mathcal{S}(G)$ such that $v$ and $w$ are duplicate vertices in $H$ and $H-w=H^{\prime}$. Apply Proposition 2.7 to $H$ and $H-w$ to yield $\operatorname{mvr}(G) \leq \operatorname{mvr}(H)=\operatorname{mvr}(H-w)=$ $\operatorname{mvr}(G-w)$. Since $G-w$ is an induced subgraph of $G, \operatorname{mvr}(G-w) \leq \operatorname{mvr}(G)$.

Remark 2.9. In Example 2.5, note that $\operatorname{mvr}\left(G_{2}-w\right)=3=\operatorname{mvr}\left(G_{2}\right)$.

Isolated and singly-isolated vertices behave differently when computing mvr. If $v$ is an isolated vertex of a graph $G$, in any non-degenerate vector representation of $G, v$ must be represented by a non-zero vector that is orthogonal to every other vector in the representation. Thus, for a simple graph $G, \operatorname{mvr}(G)=\operatorname{msr}(G)+i$, where $i$ is the number of isolated vertices [11]. This is also true for graphs with multiple edges as long as no singly-isolated vertices are involved.

Proposition 2.10. If $G$ is a multigraph that does not contain any singly-isolated vertices, $\operatorname{mvr}(G)=\operatorname{msr}(G)+i$, where $i$ is the number of isolated vertices of $G$. Further, mvr (like msr) is additive on connected components.

Proof. Both mvr and msr are additive on connected components since the spans of vectors representing different connected components must be orthogonal subspaces. The first claim follows from this given $\operatorname{mvr}\left(K_{1}\right)=1$ and $\operatorname{msr}\left(K_{1}\right)=0$, where $K_{1}$ is a single vertex, and that $\operatorname{mvr}(H)=\operatorname{msr}(H)+i$ for any $H$ in $\mathcal{S}(G)$, where $i$ is the number of isolated vertices of $G$ (and also of $H$ since $G$ has no singlyisolated vertices).

When singly-isolated vertices are present, they may or may not contribute to mvr, as shown by Example 2.11.

EXAMPLE 2.11. We can construct a graph $G$ with $n$ singly-isolated vertices where $\operatorname{mvr}(G)=\operatorname{msr}(G)+m$, for any $0 \leq m \leq n$. To do so, take a complete simple graph 


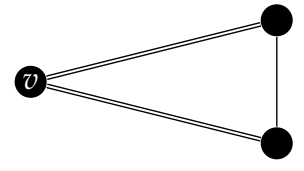

$G_{1}$

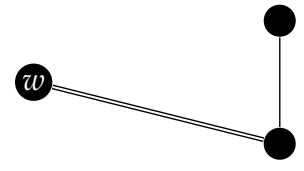

$G_{2}$

FIG. 2.3. Graphs to accompany Example 2.12.

on $2 n+1$ vertices, choose $n-m$ of those vertices and double the edges incident to them so that they become singly-isolated. Finally, take $m$ of the remaining vertices that are not singly-isolated and adjoin a singly-isolated vertex to each. The resulting graph has msr one and mvr equal to $m+1$.

A consequence of considering only non-degenerate vector representations is that if $v$ is an isolated vertex of $G$, then $\operatorname{mvr}(G)=\operatorname{mvr}(G-v)+1$. Again, however, singly-isolated vertices can behave differently, as shown in Example 2.12.

ExAmple 2.12. In the graphs $G_{1}$ and $G_{2}$ of Figure 2.3, $v$ and $w$ are singly isolated, and inspection shows that $\operatorname{mvr}\left(G_{1}\right)=\operatorname{mvr}\left(G_{1}-v\right)=1$ while $\operatorname{mvr}\left(G_{2}\right)=\operatorname{mvr}\left(G_{2}-\right.$ $w)+1$.

Proposition 2.13. Let $v$ be a singly-isolated vertex of a graph $G$. If for every simple graph $H$ in $\mathcal{S}(G)$ with $\operatorname{mvr}(G)=\operatorname{mvr}(H)$ the vertex $v$ is not isolated in $H$ then $\operatorname{mvr}(G)=\operatorname{mvr}(G-v)$. Otherwise $\operatorname{mvr}(G)=\operatorname{mvr}(G-v)+1$.

Proof. First, notice that $\operatorname{mvr}(G) \leq \operatorname{mvr}(G-v)+1$ since adjoining an isolated vertex $v$ to any $H$ in $\mathcal{S}(G-v)$ increases the mvr by one. Since $G-v$ is an induced subgraph of $G, \operatorname{mvr}(G-v) \leq \operatorname{mvr}(G)$. Suppose for every simple graph $H \in \mathcal{S}(G)$ with $\operatorname{mvr}(G)=\operatorname{mvr}(H)$ the vertex $v$ is not isolated in $H$. If $\operatorname{mvr}(G)=\operatorname{mvr}(G-$ $v)+1$, then adjoining an isolated vertex $v$ to any $H^{\prime}$ in $\mathcal{S}(G-v)$ yields a graph $H$ in $\mathcal{S}(G)$ with $\operatorname{mvr}(H)=\operatorname{mvr}(G)$ and where $v$ is isolated, contradicting our assumption. Hence, $\operatorname{mvr}(G)=\operatorname{mvr}(G-v)$. $\square$

Proposition 2.14. For any graph $G, \operatorname{msr}(G)+i \leq \operatorname{mvr}(G) \leq \operatorname{msr}(G)+i+m$, where $i$ is the number of isolated vertices of $G$ and $m$ is the number of singly-isolated vertices of $G$.

Proof. If $G$ has no singly-isolated vertices, this follows from Proposition 2.10. Assume the result is true for all graphs with $m-1$ singly-isolated vertices, and let $G$ have $m$ singly-isolated vertices. Let $v$ be a singly-isolated vertex. By Proposition 2.13, $\operatorname{mvr}(G-v) \leq \operatorname{mvr}(G) \leq \operatorname{mvr}(G-v)+1$. Since $v$ is singly-isolated, $\operatorname{msr}(G)=$ $\operatorname{msr}(G-v)$. By the induction hypothesis, $\operatorname{msr}(G-v)+i \leq \operatorname{mvr}(G-v) \leq \operatorname{msr}(G-$ 
v) $+i+m-1$, so that

$$
\begin{array}{r}
\operatorname{msr}(G)+i=\operatorname{msr}(G-v)+i \leq \operatorname{mvr}(G-v) \leq \operatorname{mvr}(G) \leq \operatorname{mvr}(G-v)+1 \\
\leq \operatorname{msr}(G-v)+i+(m-1)+1=\operatorname{msr}(G)+i+m .
\end{array}
$$

3. Simplicial Vertices. For a connected chordal graph with no singly-isolated vertices, $\operatorname{msr}(G)=\operatorname{cc}(G)$ [4] (the statement is actually for any connected chordal graph, but as an edge cover, $\mathrm{cc}(G)$ is defined to not require covering singly-isolated vertices). We now consider an analogue of this result and its corollaries for mvr. Because degenerate vector representations may be considered when computing msr, it does not matter that the orthogonal removal of a vertex from a vector representation may result in a vector representation containing zero vectors. However, when computing mvr, one may only orthogonally remove a vertex $v$ if for every nondegenerate vector representation of the graph there does not exist another vertex $w$ for which the vectors representing $v$ and $w$ are parallel. We have already noted that this condition does not imply that one vertex duplicates another. However, if the vertex $v$ is simplicial, we can say more:

Proposition 3.1. For any vertex $v$ of a graph $G, \operatorname{mvr}(G \ominus v) \leq \operatorname{mvr}(G)$. If $v$ is simplicial, then $\operatorname{mvr}(G \ominus v) \leq \operatorname{mvr}(G) \leq \operatorname{mvr}(G \ominus v)+1$. If $v$ is a simplicial vertex and there does not exist a neighbor $w$ of $v$ for which $N_{1}[w] \subseteq N[v]$, then $\operatorname{mvr}(G)=$ $\operatorname{mvr}(G \ominus v)+1$.

Proof. Note that the condition $N_{1}[w] \subseteq N[v]$ means that if $w$ is adjacent to any vertex in $G-N[v]$, then it is adjacent by multiple edges. If there exists a neighbor $w$ of $v$ for which $N_{1}[w] \subseteq N[v]$, if $\vec{V}$ is a vector representation of $G$, then $\vec{V} \ominus \vec{v}$ may not be a vector representation of $G \ominus v$. However, since for each $w_{i}$ such that $N_{1}\left[w_{i}\right] \subseteq N[v]$ in $G, w_{i}$ is singly-isolated in $G \ominus v$, and all such $w_{i}$ form a clique in $G \ominus v$, so that using Lemma 1.1 and that $\operatorname{rank}(\vec{V} \ominus \vec{v})=\operatorname{mvr}(G)-1$, we may construct a non-degenerate vector representation of $G \ominus v$ with $\operatorname{rank} \operatorname{mvr}(G)$, showing that $\operatorname{mvr}(G) \geq \operatorname{mvr}(G \ominus v)$.

If $v$ is simplicial, then $\operatorname{mvr}(G) \leq \operatorname{mvr}(G \ominus v)+1$ by Lemma 1.1. If, further, there does not exist a neighbor $w$ of $v$ for which $N_{1}[w] \subseteq N[v]$, then there are no singly isolated vertices in $G \ominus v$ and $\vec{V} \ominus \vec{v}$ is a vector representation of $G \ominus v$. As a result, $\operatorname{mvr}(G) \geq \operatorname{mvr}(G \ominus v)+1$. Together with the above, this gives that $\operatorname{mvr}(G)=\operatorname{mvr}(G \ominus v)+1$.

Using non-degenerate vector representations and without the need to assume that the vertex is not singly-isolated, the proof of Proposition 2.6 gives the following:

Proposition 3.2. Let $v$ be a simplicial vertex of a graph $G, w$ a neighbor of $v$, and 
$N_{1}[w] \subseteq N[v]$. Then $\operatorname{mvr}(G)=\operatorname{mvr}(G-w)$

Theorem 3.3. Let $G$ be a chordal graph. Then $\operatorname{mvr}(G)=\theta_{2}(G)$.

Proof. Induct on the number of vertices of $G$. If $G$ has one vertex, then $\operatorname{mvr}(G)=$ $\theta_{2}(G)=1$. For graphs with two or more vertices, since $G$ is chordal, it has a simplicial vertex $v$. If there exists a neighbor $w$ of $v$ in $G$ such that $N_{1}[w] \subseteq N[v]$, then $\operatorname{mvr}(G)=\operatorname{mvr}(G-w)$ by Proposition 3.2, $\theta_{2}(G)=\theta_{2}(G-w)$ by definition, and the result follows from applying the induction hypothesis to $G-w$. If there is no such neighbor of $v$, then $\operatorname{mvr}(G)=\operatorname{mvr}(G \ominus v)+1$ by Proposition 3.1, $\theta_{2}(G)=$ $\theta_{2}(G \ominus v)+1$, and the result follows from applying the induction hypothesis to $G \ominus v . \square$

Corollary 3.4. For a connected chordal graph $G, \operatorname{mvr}(G)-\operatorname{msr}(G)$ is exactly $\theta_{2}(G)-\theta_{1}(G)$.

4. Vector Coloring. For a simple graph $G$, let $D(G)$ be the multigraph obtained by adding another edge to $G$ wherever there already is one, and let $G^{c}$ be the usual complement of $G$. We end by considering the mvr of graphs that have the form $D(G)$ for some simple graph $G$. These are graphs for which the relationship between msr (which is zero) and mvr is at its most distant.

Recall that a graph is perfect if for all induced subgraphs $H$ of $G, \chi(H)=$ $\omega(G)$. A subset of vertices of a graph $G$ is independent if no two are adjacent. The independence number $\alpha(G)$ is the size of a largest independent set of $G$. Since the vertices of an independent set must be represented by mutually orthogonal vectors, $\operatorname{mvr}(G) \geq \alpha(G)$ for any graph $G$.

A vertex coloring of a graph $G=(V, E)$ is a function $\varphi: V \rightarrow C$ from the set of vertices to a set $C$ of colors. A coloring $\varphi$ is valid if no two adjacent vertices are assigned the same color. A $k$-coloring is a valid vertex-coloring with at most $k$ colors. A graph is $k$-colorable if it admits a valid vertex-coloring with $k$ colors. A graph is $k$-chromatic if it is $k$-colorable but not $(k-1)$-colorable, in which case the chromatic number $\chi(G)$ is $k$.

For a simple graph $G$, the parameter $\operatorname{mvr}(D(G))$ is equal to the vector chromatic number of the complement of $G$ [10]. That is, $\operatorname{mvr}(D(G))=\chi_{v}\left(G^{c}\right)$. When restricted to vectors over the real numbers, the parameter $\operatorname{mvr}\left(D\left(G^{c}\right)\right)$ was used by Lovász in his solution of the Shannon capacity of $C_{5}$ [15] and his characterization (with Saks and Schrijver) of $k$-connected graphs $[12,13]$. A related parameter was introduced and used by Haemers [8,9] to give an upper bound on Shannon capacity. See the survey by Lovász and Vesztergombi [14] for further information.

Remark 4.1. From the definitions, for all simple graphs $G, \chi(G)=\theta\left(G^{c}\right)$, 
$\alpha\left(G^{c}\right)=\omega(G)$, and $\chi_{v}(G) \leq \chi(G)$. Further, $\alpha(G)=\alpha(D(G))$ and $\omega(G)=$ $\omega(D(G))$.

Proposition 4.2. If $G$ is a simple perfect graph, then $\operatorname{mvr}(D(G))=\alpha(G)$.

Proof. Since $G$ is perfect, by the Perfect Graph Theorem [20], $G^{c}$ is perfect, and so $\chi\left(G^{c}\right)=\omega\left(G^{c}\right)$. Using Remark 4.1,

$$
\alpha(G) \leq \operatorname{mvr}(D(G))=\chi_{v}\left(G^{c}\right) \leq \chi\left(G^{c}\right)=\omega\left(G^{c}\right)=\alpha(G) .
$$

Corollary 4.3. If $G$ is a simple chordal graph, then $\alpha(G)=\operatorname{mvr}(D(G))=$ $\theta_{2}(D(G))=\theta(G)$.

Proposition 4.4. If $G$ is a simple bipartite graph, $\operatorname{mvr}(D(G))=\alpha(G)=\theta(G)$.

Proof. Combine Proposition 4.2 with the Strong Perfect Graph Theorem [6].

Most simple odd cycles are examples of graphs $G$ for which $\operatorname{mvr}(D(G)) \neq$ $\alpha(G)$.

Proposition 4.5. If $G$ is a simple path or simple cycle, then $\operatorname{mvr}(D(G))=\theta(G)$.

Proof. For a simple path, simple even cycle, or complete graph on three vertices, the result follows from Proposition 4.4 and Proposition 4.2. For a simple odd cycle $C_{n}$ on $n>3$ vertices, $\operatorname{mvr}\left(D\left(C_{n}\right)\right) \leq \theta_{2}\left(D\left(C_{n}\right)\right)=\theta\left(D\left(C_{n}\right)\right)=\left\lceil\frac{n}{2}\right\rceil$. Suppose there exists a non-degenerate vector representation of $D\left(C_{n}\right)$ with rank $\alpha\left(C_{n}\right)=\left\lfloor\frac{n}{2}\right\rfloor<$ $\left\lceil\frac{n}{2}\right\rceil$. If $S$ is a maximal independent set of vertices, then there must be a vertex $v$ adjacent to only one vertex $w$ of $S$, so that the vector representing $v$ must be a nonzero multiple of the vector representing $w$. However, this implies $v$ is adjacent to the other neighbor of $w$, a contradiction. Thus

$$
\operatorname{mvr}\left(D\left(C_{n}\right)\right)=\theta\left(D\left(C_{n}\right)\right)=\left\lceil\frac{n}{2}\right\rceil \cdot \mathrm{\square}
$$

Although difficult to find, there are examples of graphs for which $\operatorname{mvr}(D(G)) \neq$ $\theta(G)$, the smallest of which currently known is on 17 vertices [10].

\section{REFERENCES}

[1] Wayne Barrett, Hein van der Holst, and Raphael Loewy. Graphs whose minimal rank is two. Electron. J. Linear Algebra, 11:258-280 (electronic), 2004.

[2] Béla Bollobás. Modern graph theory, volume 184 of Graduate Texts in Mathematics. Springer-Verlag, New York, 1998.

[3] Matthew Booth, Philip Hackney, Benjamin Harris, Charles R. Johnson, Margaret Lay, Terry Lenker, Lon H. Mitchell, Sivaram K. Narayan, Amanda Pascoe, and Brian D. Sutton. On the minimum 
semidefinite rank of a simple graph. To appear in Linear and Multilinear Algebra.

[4] Matthew Booth, Philip Hackney, Benjamin Harris, Charles R. Johnson, Margaret Lay, Lon H. Mitchell, Sivaram K. Narayan, Amanda Pascoe, Kelly Steinmetz, Brian D. Sutton, and Wendy Wang. On the minimum rank among positive semidefinite matrices with a given graph. SIAM Journal on Matrix Analysis and Applications, 30(2):731-740, 2008.

[5] Robert C. Brigham and Ronald D. Dutton. On clique covers and independence numbers of graphs. Discrete Math., 44(2):139-144, 1983.

[6] Reinhard Diestel. Graph theory, volume 173 of Graduate Texts in Mathematics. Springer-Verlag, Berlin, third edition, 2005.

[7] Philip Hackney, Benjamin Harris, Margaret Lay, Lon H. Mitchell, Sivaram K. Narayan, and Amanda Pascoe. Linearly independent vertices and minimum semidefinite rank. Linear Algebra and its Applications, 431(8):1105 - 1115, 2009.

[8] W. Haemers. An upper bound for the Shannon capacity of a graph. In Algebraic methods in graph theory, Vol. I, II (Szeged, 1978), volume 25 of Colloq. Math. Soc. János Bolyai, pages 267-272. North-Holland, Amsterdam, 1981.

[9] Willem Haemers. On some problems of Lovász concerning the Shannon capacity of a graph. IEEE Trans. Inform. Theory, 25(2):231-232, 1979.

[10] Gerald Haynes, Lon H. Mitchell, Catherine Park, Amanda Schaeffer, and Jordan Webster. Orthogonal vector coloring. Electron. J. Combin., 17(1):R55, 2010.

[11] Yunjiang Jiang, Lon H. Mitchell, and Sivaram K. Narayan. Unitary matrix digraphs and minimum semidefinite rank. Linear Algebra Appl., 428(7):1685-1695, 2008.

[12] L. Lovász, M. Saks, and A. Schrijver. Orthogonal representations and connectivity of graphs. Linear Algebra Appl., 114/115:439-454, 1989.

[13] L. Lovász, M. Saks, and A. Schrijver. A correction: “Orthogonal representations and connectivity of graphs" [Linear Algebra Appl. 114/115 (1989), 439-454; MR0986889 (90k:05095)]. Linear Algebra Appl., 313(1-3):101-105, 2000.

[14] L. Lovász and K. Vesztergombi. Geometric representations of graphs. In Paul Erdős and his mathematics, II (Budapest, 1999), volume 11 of Bolyai Soc. Math. Stud., pages 471-498. János Bolyai Math. Soc., Budapest, 2002.

[15] László Lovász. On the Shannon capacity of a graph. IEEE Trans. Inform. Theory, 25(1):1-7, 1979.

[16] T. D. Parsons and Tomaž Pisanski. Vector representations of graphs. Discrete Math., 78(1-2):143-154, 1989.

[17] Norman J. Pullman. Clique coverings of graphs-a survey. In Combinatorial mathematics, X (Adelaide, 1982), volume 1036 of Lecture Notes in Math., pages 72-85. Springer, Berlin, 1983.

[18] Debra Taylor, Ronald D. Dutton, and Robert C. Brigham. Bounds on Nordhaus-Gaddum type bounds for clique cover numbers. In Proceedings of the fourteenth Southeastern conference on combinatorics, graph theory and computing (Boca Raton, Fla., 1983), volume 40, pages 389-398, 1983.

[19] Hein van der Holst. Graphs whose positive semi-definite matrices have nullity at most two. Linear Algebra Appl., 375:1-11, 2003.

[20] Douglas B. West. Introduction to graph theory. Prentice Hall Inc., Upper Saddle River, NJ, 1996. 